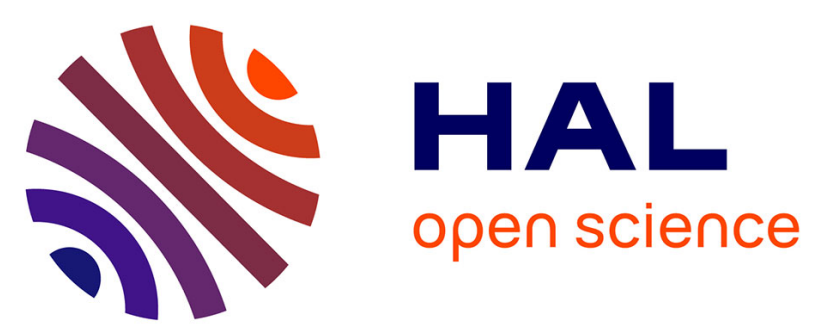

\title{
Global sensitivity analysis of large-scale numerical landslide models based on Gaussian-Process meta modelling
}

Jeremy Rohmer, Evelyne Foerster

\section{To cite this version:}

Jeremy Rohmer, Evelyne Foerster. Global sensitivity analysis of large-scale numerical landslide models based on Gaussian-Process meta modelling. Computers \& Geosciences, 2011, 37 (7), pp.917-927. 10.1016/j.cageo.2011.02.020 . hal-00578781

\section{HAL Id: hal-00578781 https://hal-brgm.archives-ouvertes.fr/hal-00578781}

Submitted on 22 Mar 2011

HAL is a multi-disciplinary open access archive for the deposit and dissemination of scientific research documents, whether they are published or not. The documents may come from teaching and research institutions in France or abroad, or from public or private research centers.
L'archive ouverte pluridisciplinaire $\mathbf{H A L}$, est destinée au dépôt et à la diffusion de documents scientifiques de niveau recherche, publiés ou non, émanant des établissements d'enseignement et de recherche français ou étrangers, des laboratoires publics ou privés. 
1 Global sensitivity analysis of large-scale numerical

2 landslide models based on Gaussian-Process meta-

\section{modelling}

Jeremy Rohmer ${ }^{1 *}$, Evelyne Foerster ${ }^{1}$

${ }^{1}$ BRGM, Natural Risks and Safety of $\mathrm{CO}_{2}$ geological Storage, 3, av. Claude Guillemin BP 36009, 45060 Orléans Cedex 2, FRANCE

e-mail:j.rohmer@brgm.fr.e.foerster@brgm.fr

\section{Abstract}

Large-scale landslide prediction is typically based on numerical modelling, with computer codes generally involving a large number of input parameters. Addressing the influence of each of them on the final result and providing a ranking procedure may be useful for risk management purposes. This can be performed by a variance-based global sensitivity analysis. Nevertheless, such an analysis requires a large number of computer code simulations, which appears impracticable for computationally-demanding simulations, with computation times ranging from several hours to several days. To overcome this difficulty, we propose a "metamodel"-based strategy consisting in replacing the complex simulator by a "statistical approximation” provided by a Gaussian-Process (GP) model. This allows computation of sensitivity measures from a limited number of simulations. For illustrative purposes, the proposed methodology is used to rank in terms of importance the properties of the

${ }^{*}$ Corresponding author: J. Rohmer, e-mail: j.rohmer@brgm.fr, Tel. + 332386430 92, Fax. +33 238643689 
elastoplastic model describing the complex behaviour of the slip surface in the La Frasse landslide (Switzerland).

One limitation of the GP-based methodology is that the computation of sensitivity measures is associated with uncertainty as the simulator is approximated using a training sample of small size, i.e. a limited knowledge on the "true" simulator. This source of uncertainty can be taken into account by treating the GP model from a Bayesian perspective. This provides the full posterior probability distribution associated with the sensitivity measures, which can be summarized by a confidence interval to outline the regions where the GP model is "unsure”. We show that this methodology is able to provide useful guidelines for the practical decisionmaking process and suggest further site investigations.

Keywords: Landslide model; computationally demanding code; global sensitivity analysis; Sobol’ indices; meta-model; Gaussian Process.

\section{Introduction}

Landslides are very complex phenomena controlled by a range of processes. Geological history, lithology and structure, slope relief and shape, weather and climate, seismicity and human activity can be identified as the main causative factors (Crosta and Clague, 2009). The associated risk to communities can be high (e.g. Evans et al., 2002) and thus, predicting landslide behaviour is a major concern.

Due to the recent advances in computer modelling (e.g. in processor performance) and in particular in the finite element method (e.g. van den Ham et al., 2009), numerical models are commonly used in practice to get a better understanding of the landslide behaviour and to predict its evolution. The main drawback of such models is the high number of input factors required for analysis. Global sensitivity analysis of complex numerical models can then be 
used to determine: (1) which input factors contribute the most to the output variability (within the “factors' prioritisation setting” as described by Saltelli et al., 2008); (2) which input factors interact with each other; and (3) which input factors are insignificant and can be eliminated to “simplify” the model (within a “factors” fixing setting”, Saltelli et al., 2008). Such an analysis is useful in identifying which input factors require further investigations to reduce uncertainties in the computer code results, hence providing guidelines for risk management (Saltelli, 2002b).

Among the existing sensitivity methods, variance-based methods have proved to be effective (Saltelli et al., 2000). In this article, we focus on the method of Sobol' indices (Sobol', 1993; Archer et al., 1997; Sobol’ and Kucherenko, 2005). Unlike traditional linear or rank regression-based methods, these indices allow representing the sensitivity of a general model without assuming any kind of linearity or monotonicity in the model (Saltelli and Sobol', 1995). In practice, the computation of Sobol' indices uses a Monte Carlo sampling strategy. An example of application in the field of landslide modelling with applications of moderate complexity is provided by Hamm et al. (2006). Such an approach, however, appears hardly applicable for more computationally demanding models, as it requires a large number of computer code evaluations. For instance, the study of Hamm et al., (2006) required ten thousand model realisations, corresponding to about 20 hours of computation time (on a 2 GHz Pentium 4 PC). The same sensitivity analysis would require 208 days using a model that takes 30 minutes and 2500 days using a model that takes 6 hours to compute.

To overcome this difficulty, a first solution is to use a distributed parallel computing methodology, thus requiring an appropriate grid computing architecture and the optimization of computing resources (e.g. Dupros et al., 2006; Boulahya et al., 2007). In this paper, an alternative is proposed using a limited number of computer code runs (also named “simulator”, O’ Hagan, 2006), which consists in replacing (i.e. approximating) the 
simulator by a surrogate model with low computation time, also named a "meta-model", to compute the Sobol' indices (i.e. the sensitivity measures). Various “meta-models” exist (e.g. linear regression, nearest neighbour method, Multivariate Adaptative Regression Spline, neural network and Gaussian Process); see, for example, Storlie et al., 2009 for a recent review.

The meta-model uses a limited number of simulator runs, i.e. input-output pairs (corresponding to the training sample), to infer the values of the complex simulator output given a yet-unseen input configuration. Such an approximation introduces a source of uncertainty referred to as “code uncertainty” associated with the meta-model (O’ Hagan, 2006), so that the sensitivity measures computed with the meta-model are "uncertain”. In the present article, we choose to solve the described problem of approximation (and of inference) under the Bayesian formalism treating the simulator as an "unknown” function in the sense that the simulator output for any yet-unseen input configuration is unknown until the simulator is actually run for the considered configuration (Oakley and O’ Hagan, 2004). We choose to use the concept of an emulator corresponding to a statistical approximation so that a prior probability distribution is assigned to the simulator outputs and updated according to the usual Bayesian paradigm given the training sample. This approach returns not only the most likely value for the output given any input configuration, but also an entire probability distribution (O’ Hagan, 2006). This distribution can be used to estimate a level of confidence when the predictive quality of the meta-model is not high due to a small training data (see, for instance, Marrel et al., 2008 and 2009, Storlie et al., 2009). A Gaussian Process (GP) is chosen as the prior model for the simulator. It has been widely used when designing computer experiments (Sacks et al., 1989; Kennedy and O’Hagan, 2001; Santner et al., 2003). In the first section, the Sobol' decomposition method is described in the general framework of the variance-based global sensitivity approach (Saltelli et al., 2008). 


$$
V_{Y}=\sum_{i} V_{i}+\sum_{i<j} V_{i j}+\sum_{i<j<l} V_{i j l}+\ldots+V_{i j \ldots n}
$$
$\mathbf{x}=\left\{x_{i}\right\}_{i=1, \ldots, n}$ so that $y=g(\mathbf{x})$.

Then, the GP model used as a meta-model of the computationally intensive simulator is described in the framework of the stochastic processes for computer code experiments under the Bayesian regression formalism. The methodology for computing the Sobol' indices using the GP model is described and illustrated in two applications. The first application is a simple analytical model based on "infinite slope analysis" (Hansen, 1984). This allows us to compare the sensitivity measures computed using the "true" model with those computed using the GP model. Finally, the application of this methodology to a La Frasse (Switzerland) landslide model (Laloui et al., 2004) is presented and we show how to use the sensitivity measures to guide the decision-making process for further site investigations.

\section{Global sensitivity analysis by the Sobol' decomposition method}

\subsection{Introduction on the variance-based sensitivity analysis}

Consider the simulator $g$ and the scalar output $y$ determined from a vector of $n$ input factors

Considering the $n$-dimensional vector as a random vector of independent random variable $X_{\mathrm{i}}$, then the output $Y$ is also a random variable (as a function of a random vector). A variancebased sensitivity analysis aims at determining the part of the total unconditional variance $V_{\mathrm{Y}}$ of the output $Y$ resulting from each input random variable $X_{\mathrm{i}}$. The total variance $V_{\mathrm{Y}}$ can be expressed as follows (Saltelli et al., 2000 \& 2008): 
$V_{i}=\operatorname{Var}\left[E\left[Y \mid X_{i}=x_{i}\right]\right]$

$V_{i j \ldots n}=\operatorname{Var}\left[E\left[Y \mid X_{i}=x_{i}, X_{j}=x_{j}, \ldots, X_{n}=x_{n}\right]\right]-V_{i}-V_{j}-\ldots-V_{n}$

119

120 with $E\left[Y \mid X_{i}=x_{i}\right]$, the expectation of $Y$ given that the $i^{\text {th }}$ input factor $X_{\mathrm{i}}$ has a fixed value $x_{\mathrm{i}}$

121 and $E\left[Y \mid X_{i}=x_{i}, X_{j}=x_{j}, \ldots, X_{n}=x_{n}\right]$ the conditional expectation of $Y$ given that the $i^{\text {th }}$ input

122 factor $X_{\mathrm{i}}$ has a fixed value $x_{\mathrm{i}}$, the $j^{\text {th }}$ input factor $X_{\mathrm{j}}$ has a fixed value $x_{\mathrm{j}}$, ...etc.

123 The variance of the conditional expectation $V_{\mathrm{i}}$ represents the first order effect of the input

124 factor $X_{\mathrm{i}}$ taken alone, whereas the higher order indices account for possible mixed influence

125 of various input factors.

126

127

\subsection{The Sobol' decomposition method}

\subsubsection{Presentation}

129 To determine the partial variances of $Y$, Sobol' (1993) proposes the following decomposition

130 of $g$ into summands of increasing dimension provided that $g$ is integrable:

131

132

$g(\mathbf{x})=g_{0}+\sum_{i=1}^{n} g_{i}\left(x_{i}\right)+\sum_{i \leq j}^{n} g_{i j}\left(x_{i}, x_{j}\right)+\ldots+g_{1, \ldots, n}\left(x_{1}, \ldots, x_{n}\right)$

133

134 where $g_{0}$ corresponds to the mean constant value of the function $g$ and each term can be

135 evaluated through multidimensional integrals as follows:

136

137

$g_{0}=E[Y]=\int_{0}^{1} \ldots \int_{0}^{1} g(\mathbf{x}) d \mathbf{x}=\int_{0}^{1} \ldots \int_{0}^{1} g\left(x_{1}, \ldots, x_{n}\right) d x_{1} \ldots d x_{n}$

138

$g_{i}\left(x_{i}\right)=E\left[Y \mid X_{i}=x_{i}\right]-g_{0}=\int_{0}^{1} \ldots \int_{0}^{1} g(\mathbf{x}) d \mathbf{x}_{-i}-g_{0}$ 
139

$$
g_{i j}\left(x_{i}, x_{j}\right)=E\left[Y \mid X_{i}=x_{i}, X_{j}=x_{j}\right]-g_{0}-g_{i}-g_{j}=\int_{0}^{1} \ldots \int_{0}^{1} g(\mathbf{x}) d \mathbf{x}_{-(i j)}-g_{0}-g_{i}\left(x_{i}\right)-g_{j}\left(x_{j}\right)
$$

140

141

with $d \mathbf{x}_{-i}$ denotes the integration over all input factors except $x_{i}$ and $d \mathbf{x}_{-(i j)}$, the integration

142 over all input factors except both $x_{\mathrm{i}}$ and $x_{\mathrm{j}}$. Similar formulae can be obtained for higher order

143 terms.

144

145 The total variance $V_{Y}$ can then be expressed as:

146

147

$V_{Y}=\int_{0}^{1} \ldots \int_{0}^{1} g\left(x_{1}, \ldots, x_{n}\right)^{2} d x_{1}, \ldots d x_{n}-g_{0}^{2}$

148

149 while the partial variances read as follows:

150

151

$$
V_{i_{1} \ldots i_{s}}=V\left[g_{i_{1} \ldots i_{s}}\right]=\int_{0}^{1} \ldots \int_{0}^{1} g_{i_{1} \ldots i_{s}}\left(x_{i_{1}}, \ldots, x_{i_{s}}\right)^{2} d x_{i_{1}} \ldots d x_{i_{s}}
$$

152 with $1 \leq i_{1}<\ldots<i_{s} \leq n$ and $s=1, \ldots, n$.

\subsubsection{Definition of the Sobol' indices}

154 The Sobol' indices $S_{i_{1} \ldots i_{s}}$ describe which amount of the total variance is due to the

155 uncertainties of input factors in the set $\left\{i_{1}, \ldots, i_{s}\right\}$ and is expressed as the ratio between $V_{i_{1} \ldots i_{S}}$

156 and $V_{Y}$, respectively the partial and total variances .

157 The first-order sensitivity index $S_{\mathrm{i}}$ for input factor $X_{\mathrm{i}}$ is expressed as follows:

158

$159 \quad S_{i}=\frac{V_{i}}{V_{Y}}$ 
160 The sensitivity measure $S_{i}$ is referred to as "the main effect of $X_{i}$ " and can be interpreted as

161 the expected reduction in the total variance of the output $Y$ (i.e. representing the uncertainty in

$162 Y$ ) if the true value of the input factor $X_{i}$ was known. This index provides a measure of

163 importance useful to rank the input factors (Saltelli et al., 2000 \& 2008).

164 The main effect and the higher order Sobol' indices satisfy the following property:

165

$166 \sum_{i=1}^{n} S_{i}+\sum_{i<j} S_{i j}+\ldots+S_{i j \ldots n}=1$

167

168 Defining $S_{-i}$ as the sum of all the terms $S_{i_{1} \ldots i_{s}}$ but the $i^{\text {th }}$ index, the total effect index $S_{T i}$ of $X_{\mathrm{i}}$

169 is defined as the total contribution of the $i^{\text {th }}$ input factor to the total variance. It reads as

170 follows (using eq. 10):

171

$172 S_{T i}=1-S_{-i}$

173

174

$S_{T i}=0$ means that the input factor $X_{\mathrm{i}}$ has no effect. Thus, it can be fixed at any value over its

175 uncertainty range (Saltelli et al., 2008).

176 As the total number of sensitivity indices reaches $2^{n}-1$ (Saltelli et al., 2000), hence

177 representing a high computational cost, the sensitivity analysis is generally limited, in practice,

178 to the pair of indicators corresponding to the main effect $S_{i}$ and to the total effect $S_{T i}$ of $X_{\mathrm{i}}$

179 (Saltelli et al., 2008).

180

\subsubsection{Numerical implementation}

181 The evaluation of the Sobol' indices can be carried out through a Monte Carlo sampling

182 strategy (Saltelli et al., 2000), which remains an approximation of the true value of the 
183

184

185

186

$$
\left\{\begin{array}{l}
\hat{g}_{0} \approx \frac{1}{m} \sum_{j=1}^{m} g\left(\mathbf{x}^{(j)}\right) \\
\hat{V}_{Y} \approx \frac{1}{m} \sum_{j=1}^{m} g^{2}\left(\mathbf{x}^{(j)}\right)-\hat{g}_{0}^{2} \\
\hat{V}_{i} \approx \frac{1}{m} \sum_{j=1}^{m} g\left(\mathbf{x}_{S_{a}}^{-(i j)}, \mathbf{x}_{S_{a}}^{(i j)}\right) \cdot g\left(\mathbf{x}_{S_{b}}^{-(i j)}, \mathbf{x}_{S_{a}}^{(i j)}\right)-\hat{g}_{0}^{2}
\end{array}\right.
$$

187

188

189

190

191

192

193

194

195

196

197

198

199 sampling data matrices are being used. and reads as follows:

sensitivity indices. Thus, the quality of the approximation directly depends on the sample size.

Let us consider $m$ sampled elements $\left\{\mathbf{x}^{(j)}\right\}_{j=1, \ldots, m}$ in the $n$-dimensional space of input factors:

where $\mathbf{x}_{-i j}=\left[x_{1 j}, \ldots, x_{(i-1) j}, x_{(i+1) j}, \ldots, x_{n j}\right]$ and the subscripts $S_{a}$ and $S_{\mathrm{b}}$ in eq. (12) indicate that two

The main and total effects can be estimated using the sampling strategy proposed by Saltelli (2002a) at a computation time cost of $m \times(n+2)$ model evaluations. Additional computational efficiency can be achieved by making best use of sampling designs, for instance Sobol' quasirandom sequences, and estimators, for instance Jansen’s estimator (Saltelli et al., 2010).

However, the computational effort for simulators with computation time ranging from several hours to several days may still be high and the present work focuses on a strategy based on Gaussian Process meta-modelling to reduce this effort.

\section{Gaussian Process (GP) modelling}

\subsection{Description of the stochastic process framework}

First, the deterministic response $\mathrm{y}(\mathbf{x})$ of the simulator is treated as a realization of a random variable $Y(\mathbf{x})$, which can be decomposed into a deterministic function $f$, which represents the mean (i.e. expectation of $Y$ ), and a stochastic function $Z$ as proposed by Sacks et al. (1989) 
206 Note that the case of multiple outputs is beyond the scope of the present article and the interested reader is advised to refer to Le and Zidek (2006).

Without prior information between the modelling inputs and outputs, $f$ is chosen as a

$$
f(\mathbf{x})=\mathbf{F}(\mathbf{x}) \cdot \mathbf{B}=\sum_{i=0}^{n} b_{i} \cdot f_{i}(\mathbf{x})
$$

213 where $\mathbf{B}=\left\{b_{i}\right\}_{i=0, \ldots, n}$ is the regression parameter vector; $\mathbf{F}(\mathbf{x})=\left[f_{0}(\mathbf{x}), f_{1}(\mathbf{x}), \ldots, f_{n}(\mathbf{x})\right]$ is the

214 corresponding regression vector with $f_{\mathrm{i}}(\mathrm{i}=0,1, \ldots, \mathrm{n})$, the basis functions. Assuming linearity

215 for the mean $f$, we have: $f_{0}(\mathbf{x})=1$ and $f_{\mathrm{i}}(\mathbf{x})=x_{i}$ for $i=1, \ldots, \mathrm{n}$.

216 The stochastic part $Z$ can be seen as a confidence measure on the model output mean. It

217 represents a zero mean random process, characterized by its $n \times n$ covariance matrix $\boldsymbol{\Sigma}_{\mathbf{S}}$ so that 218 an element at the $j^{\text {th }}$ row and $k^{\text {th }}$ column of $\boldsymbol{\Sigma}_{\mathbf{S}}$ is expressed as:

$$
\Sigma_{\mathrm{S}_{j, k}}=\operatorname{Cov}\left[Z\left(\mathbf{x}^{(j)}\right), Z\left(\mathbf{x}^{(k)}\right)\right]_{j, k=1 \ldots N_{S}}=\sigma^{2} \cdot \rho\left(\mathbf{x}^{(j)}, \mathbf{x}^{(k)}\right)_{j, k=1 \ldots N_{S}}
$$
where $\mathbf{x}^{(j)}$ is the $j^{\text {th }}$ input vector (with $j=1 \ldots N_{\mathrm{S}}$ ) and $\operatorname{Var}[\mathrm{Z}]=\sigma^{2}$. The correlation function $\rho$ provides the interpolation and spatial correlation properties.

224 Treating the simulator $g$ within the stochastic process framework allows the deterministic part $225 f$ to account for the global behaviour of $g$, whereas the correlation terms allow the meta-model 226 to "locally" interpolate the known data by introducing a strong correlation in the 
neighbourhood of these points. Complex input-output behaviours are hence better represented 228 (e.g. see Langewisch and Apostolakis, 2010).

229

230

231

232

233

234

$\rho\left(\mathbf{x}^{(j)}, \mathbf{x}^{(k)}\right)=\prod_{i=1}^{n} \rho_{i}\left(x_{i}^{(j)}, x_{i}^{(k)}\right)=\exp \left\{-\sum_{i=1}^{n} \cdot \frac{\left\|x_{i}^{(j)}-x_{i}^{(k)}\right\|^{2}}{\theta_{i}}\right\}$

where $\theta=\left\{\theta_{i}\right\}_{i=1, \ldots, \mathrm{n}}$ are the correlation lengths, also referred to as "hyper-parameters” (e.g. see

Rasmussen and Williams, 2006). $\theta_{i}$ parameter quantifies the rate at which the output varies as

$241 i^{\text {th }}$ input factor is changed.

242 Note that in case of data measurements errors or non deterministic computer code, a constant

243 regularization term referred to as "nugget effect” may be defined, hence introducing a white 244 noise.

\subsection{Principle and prediction under the Bayesian formalism}

246 In this paper, we focus on the stationary GP model which fits the stochastic framework and

247 has been broadly used in designing computer experiments (Sacks et al., 1989; Kennedy and

248 O’Hagan, 2001; Santner et al., 2003). 
249 Let us define the training sample as the $N_{S}$ training data pairs $\left\{\mathbf{X}_{S}, \mathbf{Y}_{S}\right\}$, which represent a

250 mapping between the spaces of input factors $\mathbf{x}_{S}=\left\{\mathbf{x}^{(j)}\right\}_{j=1 \ldots N_{S}}$, with $\mathbf{x}^{(j)}=\left\{x_{i}^{(j)}\right\}_{i=1, \ldots, n}$, and the 251 outputs $\mathbf{Y}_{S}=\left\{y^{(j)}\right\}_{j=1 \ldots N_{S}}$, obtained through the $N_{S}$ selected simulator runs so that $252 \quad y^{(j)}=\left\{f\left(\mathbf{x}^{(j)}\right)\right\}_{j=1, \ldots, N_{S}}$.

253 In a first step, constructing the GP model implies considering the simulator output $Y$ as a

254 random variable, which is assumed to follow a multivariate Gaussian distribution (denoted $G$ )

255 for any random vector of input factors $\mathbf{X}$. This assumption represents our prior belief on the 256 simulator. Using the training sample $\left\{\mathbf{X}_{S}, \mathbf{Y}_{S}\right\}$, the Bayes theorem is used to refine the

257 mentioned prior information in order to yield the posterior distribution of the output, known 258 as the “emulator” (e.g. O’ Hagan, 2006). This latter not only provides an expected value for 259 any “yet-unseen” input configuration, but it also gives an entire posterior distribution given 260 the observed data.

261 Formally, the probability $p\left(\mathbf{Y}_{S} \mid \mathbf{X}_{S}\right)$ of obtaining $\mathbf{Y}_{S}$ given $\mathbf{X}_{S}$, is expressed under the GP

262 assumption, as follows:

$263 p\left(\mathbf{Y}_{S} \mid \mathbf{X}_{S}\right) \sim G\left(F\left(\mathbf{X}_{S}\right) \cdot \mathbf{B}, \mathbf{\Sigma}_{S}\right)$

264 Considering a new vector $\mathbf{x}^{\left(N_{S}+1\right)}$ of input factors and the associated output $Y^{\left(N_{S}+1\right)}=Y\left(\mathbf{x}^{\left(N_{S}+1\right)}\right)$, 265 the joint probability distribution of the random variables $\left(\mathbf{Y}_{S}, Y^{\left(N_{S}+1\right)}\right)$ reads as follows:

$$
p\left(\mathbf{Y}_{S}, Y^{\left(N_{S}+1\right)} \mid \mathbf{X}_{S}, \mathbf{B}, \sigma, \boldsymbol{\theta}\right) \sim G\left(\left[\begin{array}{c}
\mathbf{F}_{S} \\
F\left(\mathbf{x}^{\left(N_{S}+1\right)}\right)
\end{array}\right] \cdot \mathbf{B},\left[\begin{array}{cc}
\boldsymbol{\Sigma}_{S} & k\left(\mathbf{x}^{\left(N_{S}+1\right)}\right) \\
k\left(\mathbf{x}^{\left(N_{S}+1\right)}\right)^{T} & \sigma^{2}
\end{array}\right]\right)
$$

267 where $\mathbf{F}_{S}=\left[F\left(\mathbf{x}^{(1)}\right), F\left(\mathbf{x}^{(2)}\right), \ldots, F\left(\mathbf{x}^{\left(N_{S}\right)}\right)\right]$ corresponds to the regression matrix.

$268 k\left(\mathbf{x}^{\left(N_{S}+1\right)}\right)$ represents the vector of correlation functions between each of the $N_{\mathrm{S}}$ training input 269 vectors $\mathbf{x}^{(j)}$ and the new element $\mathbf{x}^{\left(N_{S}+1\right)}$. It can be written as: 
273 Within the Bayesian framework, the posterior distribution of the computed output random

274 variable $Y^{\left(N_{S}+1\right)}$ is conditioned on the "observed” (i.e. actually calculated) values

275 corresponding to the training sample $\left\{\mathbf{X}_{S}, \mathbf{Y}_{S}\right\}$, given the new element $\mathbf{x}^{\left(N_{S}+1\right)}$, and follows a 276 multivariate Gaussian distribution (Von Mises, 1964):

277

278

$$
\begin{aligned}
p\left(Y^{\left(N_{S}+1\right)} \mid \mathbf{Y}_{S}, \mathbf{X}_{S}, \mathbf{B}, \boldsymbol{\sigma , \boldsymbol { \theta }}\right) & =\frac{p\left(\mathbf{Y}_{S}, Y^{\left(N_{S}+1\right)} \mid \mathbf{X}_{S}, \mathbf{B}, \boldsymbol{\sigma}, \boldsymbol{\theta}\right)}{p\left(\mathbf{Y}_{S} \mid \mathbf{X}_{S}, \mathbf{B}, \sigma, \boldsymbol{\theta}\right)} \\
& \sim G\left(E\left[Y^{\left(N_{S}+1\right)} \mid \mathbf{Y}_{S}, \mathbf{X}_{S}, \mathbf{B}, \boldsymbol{\sigma}, \boldsymbol{\theta}\right] \operatorname{Var}\left[Y^{\left(N_{S}+1\right)} \mid \mathbf{Y}_{S}, \mathbf{X}_{S}, \mathbf{B}, \sigma, \boldsymbol{\theta}\right]\right)
\end{aligned}
$$

where:

$E\left[Y^{\left(N_{S}+1\right)} \mid \mathbf{Y}_{S}, \mathbf{X}_{S}, \mathbf{B}, \sigma, \boldsymbol{\theta}\right]=F\left(\mathbf{x}^{\left(N_{S}+1\right)}\right) \cdot \mathbf{B}+k\left(\mathbf{x}^{\left(N_{S}+1\right)}\right)^{T} \cdot \boldsymbol{\Sigma}_{S}^{-1} \cdot\left(\mathbf{Y}_{S}-\mathbf{F}_{S} \cdot \mathbf{B}\right)$

281 and:

282

$$
\operatorname{Var}\left[Y^{\left(N_{S}+1\right)} \mid \mathbf{Y}_{S}, \mathbf{X}_{S}, \mathbf{B}, \sigma, \boldsymbol{\theta}\right]=\sigma^{2}-k\left(\mathbf{x}^{\left(N_{S}+1\right)}\right)^{T} \cdot \mathbf{\Sigma}_{S}^{-1} \cdot k\left(\mathbf{x}^{\left(N_{S}+1\right)}\right)
$$

with $\boldsymbol{\Sigma}_{S}^{-1}$, the inverse of the covariance matrix associated to the training input data $\mathbf{X}_{S}$.

The conditional mean of eq. (18b) is used as a predictor and the conditional variance in eq. (18c) corresponds to the mean square error of the predictor term. Provided that the new candidate $\mathbf{x}^{\left(N_{S}+1\right)}$ is far away from the training input data $\mathbf{X}_{S}$, the term $k\left(\mathbf{x}^{\left(N_{S}+1\right)}\right)^{T} \cdot \mathbf{\Sigma}_{S}^{-1} \cdot k\left(\mathbf{x}^{\left(N_{S}+1\right)}\right)$ will be small so that the predicted variance will be large. In a more general manner, if we consider two new test candidates $\mathbf{u}$ and $\mathbf{v}$, the general expression of the conditional GP model can be written as: 
294 The conditional mean is used as a predictor and is expressed as in eq. (18b).

295 The conditional covariance provides the confidence on the prediction and reads as follows:

$$
\operatorname{Cov}\left[Y(\mathbf{u}), Y(\mathbf{v}) \mid \mathbf{Y}_{S}, \mathbf{X}_{S}, \mathbf{B}, \sigma, \boldsymbol{\theta}\right]=\sigma^{2} \rho(\mathbf{u}, \mathbf{v})-k(\mathbf{u})^{T} \cdot \mathbf{\Sigma}_{S}^{-1} \cdot k(\mathbf{v})
$$

The regions of the input space, where few data are available will be underlined by higher variance.

The main difficulty in constructing a conditional GP model given a training sample resides in determining the parameters corresponding to the regression coefficient vector $\mathbf{B}$, the hyperparameters $\theta$ and the variance $\sigma^{2}$. A first approach consists in estimating them as solutions of the optimization problem using the maximum likelihood method, e.g. implemented in the GEM-SA software (O’Hagan, 2006) and the MATLAB toolbox DACE (Lophaven, 2002). However, the optimisation algorithms used for the parameters identification may show

307 limitations, especially in case of high dimension problem (e.g. see Marrel et al., 2008).

308 Besides, such an approach may underestimate the variance in the predictions of new

309 observations (Cressie, 1993).

310 In this paper, an approach based on the Bayesian framework (e.g. Rasmussen 1996) is chosen so that the hyper-parameters are given prior distributions $p(\theta)$. In the Bayesian framework, the

313 posterior distribution $p\left(\boldsymbol{\theta} \mid \boldsymbol{X}_{\mathrm{S}}, \mathbf{Y}_{\mathrm{S}}\right)$ associated with the GP parameters i.e. the training sample is 314 used to update the GP parameters. The posterior distribution of the hyper-parameters will be 315 hence concentrated on values that are consistent with actually observed data. This procedure 316 is implemented in the package named "TGP" of the "R" software ("R" Development Core 
Team, 2009) by Gramacy and co-workers (Gramacy, 2007; Gramacy and Taddy, 2010).

318 Further theoretical details can be found in Gelman et al. (1995) and Gramacy and Herbert

319 (2009). Though computationally more intensive (Storlie et al., 2009), this approach presents

320 the attractive feature to incorporate the uncertainty related to the construction of the GP model,

321 so that the level of confidence associated with the "meta-model”-computed sensitivity

322 measures also takes this source of uncertainty into account (see step 4 of section 3.4.).

\section{$323 \quad 3.4$ A “GP-based” methodology for sensitivity analysis}

324 In this section, we describe the methodology to compute the sensitivity measures (i.e. the

325 Sobol' indices) using a GP model as a surrogate model of the computationally intensive 326 simulator.

\subsubsection{Step 1: representation of the input factor uncertainty}

The first step is to characterize and mathematically represent the uncertainty (range and form of the probability distribution) on each of the input factors. This representation can have a strong influence on the final sensitivity results, hence on risk management decision making

331 (Saltelli, 2002b).

Representing the uncertainty through empirical probability distributions requires a large amount of data (laboratory or in situ measurements), which may not be practical in many situations. Thus, knowledge on the range of uncertainty is commonly evaluated either based on physical reasoning, on analogies with similar cases or simply from expert opinions, whereas the mathematical representation of the probability distribution may either be theoretically known or assumed. In a situation where "sparse, vague and incomplete" data are available, a common approach consists in assigning a uniform probability distribution based on the "maximum entropy" approach (Gzyl, 1995). 


\subsubsection{Step 2: setting training data}

341 The objective then is to run the simulator for a limited number of times $N_{\mathrm{S}}$ in order to create a mapping between the input factor and the computer code output domain. The number $N_{\mathrm{S}}$

343 should be defined as a compromise between the minimization of the computation time cost

344 and the maximization of the input factor domain exploration (directly linked with the

345 accuracy and reliability of the GP model, see step 3).

346 In this view, we propose to use the Latin hypercube sampling method (McKay et al., 1979) in 347 combination with the "maxi-min” space-filling design criterion (Koehler and Owen, 1996).

348 More sophisticated strategies exist mainly based on sequentially adaptive design of

349 experiments adding new training candidates where the predictive uncertainty is high (e.g.

350 Gramacy and Herbert, 2009). The use of such approaches is beyond the scope of this paper.

\subsubsection{Step 3: constructing the GP model}

Using the GP model instead of the simulator introduces an additional source of uncertainty referred to as “code uncertainty” (O’ Hagan, 2006). In the regions where the true simulator is not evaluated, we are uncertain about what the "true" simulator would introduce. This sort of uncertainty can be reduced by increasing our knowledge of the true simulator, i.e. by increasing the training sample size.

Except when a "nugget” effect is included, the GP model is an exact interpolator, so that residuals of the training data cannot be directly used to validate the approximation (Marrel et $a l .$, 2008). The key aspect for validating the "statistical" approximation is to estimate the expected level of fit (i.e. predictive quality) of the GP model to a data set that is independent of the data (i.e. “yet-unseen” data) that were used to train the GP model.

As additional simulator runs are costly, using a test sample of new data might be impractical

363 and cross-validation procedures such as the “ $k$-fold” cross-validation technique (Hastie, 2002)

364 should be used. In this cross-validation procedure, the initial training sample is randomly 
partitioned into $k$ subsets. In a first step, a single subset is used as the validation data for testing the GP model, and the remaining $k$-1 subsets are used as training data for the construction of the GP model. For each step, the $k$ validation data are estimated and the coefficient of determination $\mathrm{R}^{2}$ for the procedure is computed as follows:

$$
R^{2}(\mathbf{y}, \hat{\mathbf{y}})=1-\frac{\sum_{i=1}^{k}\left(\hat{y}_{i}-y_{i}\right)}{\sum_{i=1}^{k}\left(y_{i}-\overline{\mathbf{y}}\right)}
$$

where $\mathbf{y}$ represents the vector of observations in the validation set; $\overline{\mathbf{y}}$ is the mean of the corresponding sample and $\hat{\mathbf{y}}$, the vector of predicted values using the GP model.

The coefficient $\mathrm{R}^{2}$ provides a metric of the predictive quality so that a value close to $100 \%$ indicates that the GP model is successful in matching the validation data. A typical threshold of $80 \%$ is commonly used to qualify the predictive quality as "satisfactory" (e.g. Marrel et al., 2008).

The cross-validation process is then repeated $k$ times using each of the $k$ subsets as validation samples. For small training sets, the cross validation procedure with $k=1$ is usually used corresponding to the so-called "leave-one-out” cross validation procedure.

\subsubsection{Step 4: estimating the sensitivity measures}

The most likely value $\mu\left(S_{i}\right)$ for the sensitivity measures is computed using the conditional mean of the GP model in eq. 18b. Additional useful information for risk management purposes is the level of confidence (or accuracy) related to the sensitivity analysis based on the GP model. A confidence interval $C I\left(S_{i}\right)$ can be defined with bounds corresponding to the $5 \%$ and to the $95 \%$ quantile of the full posterior distribution of the sensitivity measures. This confidence interval both summarizes the "code uncertainty" associated with the meta-model (O’ Hagan, 2006) and the uncertainty on the estimation of the GP model parameters (see section 3.3). 


\section{Illustrative analytical model}

389 In this section, we consider the infinite slope analytical model (e.g. Hansen, 1984) in order to

390 illustrate the methodology described in section 3.4. This model is of course not a

391 computationally demanding function, but we imagine it as representing a calculation that may

392 take several minutes or even hours of computation to evaluate. Besides, using this analytical

393 model also allows us to compare the results of the sensitivity analysis using the "true" model

394 with those using the GP model.

\subsection{Description of the analytical model}

396 The stability of the infinite slope model as depicted in Fig. 1 is evaluated by deriving the

397 factor of safety $F S$, which corresponds to the ratio between the resisting and the driving forces

398 acting on the slope (eq. 22):

399

400

$F S=\frac{C+\left(\gamma-m \cdot \gamma_{w}\right) \cdot z \cdot \cos (\theta) \cdot \cos (\theta) \cdot \tan (\phi)}{\gamma \cdot z \cdot \sin (\theta) \cdot \cos (\theta)}$

401

402

[Fig. 1 about here]

403

404 where $C$ is the cohesion of the soil material; $\phi$, the friction angle; $\theta$, the slope angle; $\gamma$, the

405 soil unit weight; $\gamma_{w}$, the water unit weight; $z$, the thickness of slope material above the slip

406 plane; and $m$, the ratio between thickness of surficial saturated slope material and z. If $F S$ is

407 lower than 1.0 the potential for failure is high. 


\subsection{Sensitivity analysis of the analytical model}

409 For illustrative purposes, we only considered the thickness $z$ and the slope angle $\theta$ as

410 uncertain input factors. The other input factors were assumed fixed: $C=10 \mathrm{kPa}, \phi=25^{\circ}$,

$411 \gamma=22 \mathrm{kN} \cdot \mathrm{m}^{-3}, \gamma_{\mathrm{w}}=9.81 \mathrm{kN} \cdot \mathrm{m}^{-3}$ and $m=90 \%$.

412 The objective was to identify whether $z$ or $\theta$ contributes the most to the $F S$ variability within a

413 “factors’ prioritisation setting”. It is assumed that very sparse data are available to

414 characterize the uncertainty on these input factors so that $z$ uniformly varies between 5 and 25

$415 \mathrm{~m}$ and $\theta$ uniformly varies between $25^{\circ}$ and $35^{\circ}$ (step 1 ). We generated two different training

416 samples of respectively 6 and 20 training data of the form $\{z ; \theta ; F S\}$, using the Latin

417 hypercube sampling approach (step 2) and for each training sample, a GP model was

418 constructed.

[Fig. 2 about here]

Fig. 2 (top) shows the comparison between the values of FS obtained from direct simulations on a grid of $10 \times 10$ in the input factor domain [5;25]×[25;35] (straight line) and from the prediction on the same grid using the GP model (dashed line) for both training samples (Fig. 2, left for 6 training data and right for 20 training data). The coefficient of determination or goodness of fit (eq. 22) estimated for both GP models was equal to $90.9 \%$ for the first training sample and to $98.8 \%$ for the second one, hence showing a very good match for both meta-models. The quality of the approximation was then estimated through a "leave-one-out" cross validation procedure (step 3): we obtained a coefficient of determination of $96.2 \%$ for the first sample and $99.7 \%$ for the second one, hence indicating a "high” predictive quality.

431 The estimated FS using both GP models (Fig. 2, middle) were compared to the "true" observed FS. The closer the dots to the straight black line, the better the approximation. 
433

434

435

436

437

438

439

440

441

442

443

444

445

446

447

448

449

450

451

452

453

The results for the computation of the main effects required (step 4)

$m \times(n+2)=2500 \times(2+2)=10000$ model evaluations using the sampling strategy of Saltelli

(2002a). The most likely of the main effects calculated with both GP models (blue dots in Fig.

2, bottom) were compared to the main effects obtained from direct simulations (red dots on

Fig. 2, bottom) by means of the R package "sensitivity” and the function referred to as

Sobol2002 (available at http://cran.r-project.org/web/packages/sensitivity/index.html). These results are summarized in Table 1.

We see that differences are larger for the GP model constructed with the lower training sample size but, however, the "true” values for the main effects still lie within the confidence interval bounded by the $5 \%$ and the $95 \%$ quantile (black cross-type marker in Fig. 2, bottom). Not surprisingly, increasing the number of training samples (i.e. our knowledge of the true function) decreases the range of code uncertainty as well as the differences between the true values and estimates (Table 1).

\section{Computationally intensive numerical model}

In this section, we present the application of the proposed GP-based sensitivity analysis methodology (section 3.4.) to the landslide finite-element model originally used for the simulation of the La Frasse (Switzerland) landslide during the 1994 crisis period (Laloui et al., 2004).

\subsection{General description of the landslide model}


457 La Frasse landslide reaches 73 million $\mathrm{m}^{3}$. Since 1975, a constant movement has been 458 observed in its upper and central parts, varying between $10.10^{-2}$ and $15.10^{-2} \mathrm{~m}$ per year 459 (Noverraz and Bonnard, 1988; Bonnard et al., 1995). The evolution of the groundwater table 460 is considered to be at the origin of the sliding and the instabilities were mainly observed 461 during the 1994 crisis (over a period of nearly 300 days). Therefore, in order to assess the 462 effect of the hydraulic regime on the geomechanical behaviour of the landslide, finite-element 463 simulations considering a 2D cross-section through the centre of the landslide were performed by Laloui et al. (2004) using the finite element program GEFDYN (Aubry et al., 1986).

465
The model is composed of 1694 nodes, 1530 quadrangular elements, and six soil layers derived from the geotechnical investigations. Fig. 3 gives an overview of the model, as well as the boundary conditions used for analysis. Instabilities observed in 1994 were triggered by pore pressure changes occurring at the base of the slide (see Laloui et al., 2004 for further details).

\section{[Fig. 3 about here]}

The general behaviour of the landslide is strongly correlated to the properties of the slip surface. The complex behaviour of the slip surface material was modelled using the Hujeux elastoplastic multi-mechanism constitutive model (Aubry et al., 1982; Hujeux, 1985; LopezCaballero et al., 2007; Lopez-Caballero and Modaressi Farahmand-Razavi, 2008) and the Mohr-Coulomb criterion was assumed for the other soil materials.

The Hujeux constitutive model permits coverage of a large range of deformation and takes into account: (1) the influence of confinement and stress path on the moduli; (2) the effects of over-consolidation; and (3) the influence of the void ratio. It can be used for granular as well as clayey soil behaviours and it is based on a Coulomb type failure criterion and the critical 
state concept. The volumetric and deviatoric hardening regimes implemented in the Hujeux model lead to a dependence on the consolidation pressure as in the Cam-Clay family models, and to the evolution of the plastic yield surface with the deviatoric and volumetric plastic strains. Moreover, the model accounts for dilatancy/contractance of soils and non-associated flowing behaviour with evolution of the plastic strain rate through a Roscoe-type dilatancy rule.

As outlined by Laloui et al. (2004), the main parameters for the slip surface materials are: (1) the bulk $(K)$ and shear $(G)$ elastic modules, which are assumed to depend on the mean effective stress through a power-type law of exponent $n_{\mathrm{e}}$; (2) the critical state and plasticity parameters, essentially the friction angle $\phi$ at perfect plasticity, the plastic compressibility $\beta$; and (3) the dilatancy angle $\Psi$, appearing in the flow rule and defining the limit between soil dilatancy and contractance.

Note that these parameters can be directly measured from either in situ or laboratory test results (Lopez-Caballero et al., 2007; Lopez-Caballero and Modaressi Farahmand-Razavi, 2008). The other Hujeux law parameters, appearing in the flow rule, the hardening and the threshold domains definition are categorized as “not-directly measurable” (Lopez-Caballero and Modaressi Farahmand-Razavi, 2008) and are estimated through numerical calibration techniques between the observed/experimental data and the simulated ones.

\subsection{Sensitivity analysis using the GP-based methodology}

The sensitivity analysis using the GP-based methodology (see section 3.4.) was carried out to assess the importance of the input factors of the Hujeux constitutive model describing the slip surface behaviour within a "factors' prioritisation setting”, so that the main effects (first order Sobol' indices) were used for ranking.

The quantity of interest was chosen as the horizontal displacement calculated at two observation points, namely in the upper (observation point 1, Fig. 3), and lower parts of the 
landslide (observation point 2, Fig. 3). The sensitivity analysis was carried out in a dynamic manner at each step of the 300 days long crisis period (decomposed into a hundred time steps). It was focused on the main measurable parameters of the Hujeux constitutive model (total number of seven input factors), the others being kept constant i.e. treated with "no uncertainty”. The properties of the other soil layers were assumed to be constant as well.

\subsubsection{Step 1: representation of the input factor uncertainty}

In this illustrative study, our objective was to explore the situation where the same "level of uncertainty” is associated with the parameters of Hujeux model: a $25 \%$ variation around the original values identified by Laloui and co-authors (Laloui et al., 2004) was affected to each of the seven input factors (Table 2). Considering no further information on the uncertainty, a uniform probability distribution was assigned to each of these input factors (see section 3.4.1.).

\subsubsection{Step 2: setting training data}

[Table 2 about here]

A total number of 30 input parameter configurations was generated. The resulting horizontal displacements computed over the crisis period are shown on Figure 4 for the observation points 1 and 2 . For a given input configuration, a simulator run required $\approx 96$ hours on a computer unit (CPU) with a $2.6 \mathrm{GHz}$ dual core processor and $1 \mathrm{~GB}$ of RAM. The training sample was generated using a grid computing architecture or computer cluster composed of $30 \mathrm{CPU}$, so that all simulations were performed in parallel. 


\subsubsection{Step 3: constructing the GP model}

532 At each step of the 1994 crisis period, a GP model was constructed using the 30 training data

533 to approximate the horizontal displacements at the observation points 1 and 2.

534 A “leave-one-out cross-validation” procedure was carried out for each step in order to assess 535 the predictive quality of the GP models. Fig. 5 depicts the temporal evolution of the 536 coefficient of determination $\mathrm{R}^{2}$ for the cross-validation procedure.

537 During the first half of the crisis period (first 150 days), $\mathrm{R}^{2}$ decreases over time for both 538 observation points between $99.9 \%$ and $\approx 95 \%$, hence indicating that the predictive quality is 539 "high" over this period. During the second half of the crisis period, the quality is still 540 satisfactory if we consider observation point 2 ( $\mathrm{R}^{2}$ varying between $\approx 80 \%$ and $\approx 95 \%$, see 541 Marrel et al., 2008), whereas it can be qualified as "low to moderate” for observation point 1 542 ( $\mathrm{R}^{2}$ steeply decreasing from $\approx 95 \%$ to $\approx 62 \%$ ), hence indicating possibly high uncertainty on 543 the GP model.

\subsubsection{Step 4: estimating the sensitivity measures}

548 The main effects were calculated using the sampling strategy of Saltelli (2002a), hence

549 requiring $m \times(n+2)=1000 \times(7+2)=9000$ GP model evaluations. Preliminary convergence tests

550 were carried out for $m=250,500,1000$ and 2000: they showed that $m=1000$ yields satisfactory

551 convergence of the sensitivity measures to two decimal places (+/- 0.025).

552 The total computation time of the GP-based sensitivity analysis reached a total of 108 hours

553 (4.5 days), including the generation of the training sample ( $\approx 4$ days), the construction of a GP 
model at each step of the crisis period $(\approx 3$ hours) and the cross-validation procedure $(\approx 3$ hours).

If the same analysis had been undertaken by direct simulations, the total computation time would have reached 9000/30×96=28800 hours (1200 days) using the same 30 CPU cluster. To achieve a computation time of 108 hours, a computer cluster composed of 8000 CPU would have been required.

\subsubsection{Analysis of the temporal evolution of the main effects}

561

562

Fig. 6 (top) depicts the temporal evolution of the "first most important” input factor (straight green line) at the observation point 1 in the upper part of the landslide (Fig. 6, left) and at the observation point 2 in the lower part of the landslide (Fig. 6, right). Similarly, Fig. 6 (bottom) provides the temporal evolution of the "second most important" input factor. The input factors (Table 2) were ranked in terms of importance based on the mean of the main effect (blue straight line, Fig. 6) computed with the GP models constructed at each instant of the crisis period.

[Fig. 6 about here]

This preliminary ranking of the input factors, only based on the mean of the main effect, was assessed again in a second step taking into account the range of uncertainty associated to the sensitivity measures i.e. using the $5 \%$ and to the $95 \%$ quantile of the posterior probability distribution associated to the main effects (black dashed line, Fig. 6). The procedure consisted in qualifying the GP model as "unsure” with respect to the sensitivity measures in regions where the confidence intervals of the first and second most important input factors intersect. Considering the observation point 1, Fig. 6 (left) shows that for the first 150 days, coefficient $n_{\mathrm{e}}$ can be identified as the "first most important" input factor with a mean of the main effect 
constant at $\approx 20 \%$, whereas the dilatancy angle $\Psi$ can be identified as the "second most

580 important” input factor with a mean of the main effect constant at $\approx 10 \%$. For the second 581 crisis period, the confidence intervals intersect and the ranking is "unsure”. Fig. 7 (left) gives 582 the mean of the main effects and the associated confidence intervals at three different steps of 583 the crisis period, namely 30 days (Fig. 7, top), 150 days (Fig. 7, middle) and 210 days (Fig. 7, 584 bottom). At 30 days, $n_{\mathrm{e}}$ can clearly be identified as the first most important input factor, but 585 the ranking of the other input factors is hardly feasible considering the intersecting confidence 586 intervals. Over time (at 150 and 210 days), the confidence intervals for all input factors 587 intersect so that the ranking is "unsure". This result is in agreement with the low coefficient of 588 determination of the cross-validation procedure over the second half of the crisis period (Fig. 5, black dashed line). As a conclusion, the knowledge on the "true" simulator should be increased for the second crisis time period in order to increase the predictive quality of the GP 591 model, hence to narrow the width of the confidence interval.

Considering the observation point 2, Fig. 6 (right) shows that before $\approx 50$ days, the confidence intervals intersect and the ranking is "unsure". Over the time period after $\approx 50$ days, coefficient $n_{\mathrm{e}}$ can be identified as the "first most important" input factor with a mean of the main effect increasing from $\approx 20 \%$ to $\approx 45 \%$, whereas the plastic compressibility $\beta$ can be identified as the "second most important" input factor with a mean of the main effect approximately constant and equal to $15 \%$. As for point 1 , Fig. 7 (right) gives the mean of the main effects and the associated confidence intervals for steps 30 days (Fig. 7, top), 150 days

602 (Fig. 7, middle) and 210 days (Fig. 7, bottom). It shows that over time, $n_{\mathrm{e}}$ and $\beta$ can be 
604150 and 210 days, but the ranking of the other input factors is hardly feasible considering the 605 intersecting confidence intervals.

606 Despite the limited number of simulator runs (30) i.e. the limited knowledge on the "true"

607 simulator, several conclusions can still be drawn to guide future investigations. The sensitivity 608 analysis based on the GP modelling emphasizes coefficient $n_{\mathrm{e}}$ as the "most important" i.e. as 609 the input factor requiring further investigations over the crisis period, whatever the part of the 610 landslide (upper or lower). In practice, the estimation of this parameter is strongly dependent 611 on the availability of lab tests at small strains, where the behaviour is truly elastic (e.g. strains 612 lower than $10^{-4}$ ). This condition is not realized for classical triaxial tests where the accuracy is 613 not better than $10^{-3}$ (e.g. Biarez and Hicher, 1994) so that this parameter is usually deduced 614 using standard values estimated for analogous types of soil. Nevertheless, such an analogy615 based approach is hardly achievable in the La Frasse landslide case as the considered soil 616 material, being on the slip surface, is inherently heterogeneous.

617 The sensitivity analysis also outlines the plastic compressibility $\beta$ as “important” for further 618 investigations in the lower part of the landslide i.e. where the evolution of pore pressures was 619 the most important. In practice, this parameter can be obtained from oedometer tests. No 620 further conclusions can be drawn without increasing the knowledge on the "true" simulator, 621 for the third (or lower) "most important input factor” due to the uncertainty on the GP model.

622 These conclusions are valid for the considered illustrative case especially regarding the

623 assumptions on the range of uncertainty assigned to all input factors (variation in a range of $62425 \%$ around the original values). Within a procedure aiming at calibrating the observed 625 displacements with the simulated ones, the uncertainty on each input factor should be 626 adequately represented making use of any kind of information related to the measurement 627 procedure of the constitutive model parameters (number of samples, estimation of 
measurement error, possibility to construct empirical probability distribution, error of

629 calibration between observed and simulated curves, etc.).

\section{Concluding remarks and further works}

631 Landslide numerical modelling involves a large number of input factors, whose influence and

632 importance should be assessed to guide risk management and possible further investigations

633 (laboratory or in situ). A variance-based global sensitivity analysis (Saltelli et al., 2008) using

634 the calculation of Sobol' indices (Sobol', 1993; Archer et al., 1997; Sobol' and Kucherenko,

635 2005) can provide such guidelines. Nevertheless, such an analysis requires a large number of

636 direct simulations (i.e. simulator runs), which can be unfeasible in practice for

637 computationally intensive models (i.e. those characterized by computation times ranging from

638 several hours to several days). In this paper, we proposed a methodology based on Gaussian

639 Process meta-modelling to perform such an analysis using a limited number of training

640 samples.

641 The construction of the training sample is based on a space-filling approach using Latin

642 Hypercube sampling. The possible correlation between input factors is not tackled in this

643 paper and this can be further developed using, for instance, the works of Hamm and co-

644 workers (Hamm et al., 2006). We presented the construction of the meta-model and how to

645 combine it with a strategy to verify the predictive quality based on a cross-validation

646 procedure. This methodology is demonstrated on a numerical model of La Frasse

647 (Switzerland) landslide (Laloui et al., 2004), where the importance of the main constitutive

648 model parameters describing the slip surface material behaviour is assessed. Due to high

649 computational costs, the GP model is constructed only using 30 simulator runs, i.e. with a

650 limited knowledge of the "true" simulator. This induces an additional source of uncertainty

651 (referred to as code uncertainty of the meta-model) on the sensitivity measures, which is 
652

653

654

655

656

657

658

659

660

661

662

663

664

665

666

667

668

tackled by treating the GP model from a Bayesian perspective: the full posterior probability distribution associated with the sensitivity measures is computed and summarized by a confidence interval used to outline the regions where the GP model is "unsure” with respect to the sensitivity measures. When a large number of input factors $(>30)$ are present, the Bayesian treatment of the GP model may show limitations as it is more computationally demanding compared to other meta-model techniques (Storlie et al., 2009). However, recent works (e.g. Marrel et al., 2009) pertaining to variable selection for GP model can be used to overcome this difficulty. In the identified "unsure” regions, further simulator runs should be carried out and the choice of the new input configurations can be guided by taking advantage of the recent advances in adaptive design of experiments (e.g. Gramacy and Herbert, 2009), which constitutes a possible future direction.

\section{Acknowledgements}

This work was funded under the BRGM's Directorate of Research project VULNERISK. The application is based on the finite-element landslide model built by the LMS of EPFL. We are grateful to B. Gramacy for useful discussion on the hyper-parameters estimations of the GP model. We would like to thank Dr. J. Douglas for proofreading and the two anonymous reviewers for their detailed and constructive reviews.

\section{References}

Archer, G.E.B., Saltelli, A., Sobol', I.M., 1997. Sensitivity measures, ANOVA like techniques and the use of bootstrap. Journal of Statistical Computation and Simulation 58, 99-120. d'analyse du comportement mécanique des sols par éléments finis avec prise en compte du couplage sol-eau-air (Gefdyn software, Finite element analysis of soil 
mechanical behaviour taking into account the soil-water-air coupling). Report of Ecole Centrale Paris (in French).

678

679

680

681

682

683

684

685

686

687

688

689

690

691

692

693

694

695

696

697

698

Aubry, D., Hujeux, J.-C., Lassoudière, F., Meimon, Y., 1982. A double memory model with multiple mechanisms for cyclic soil behaviour. In: Proceedings of the International symposium on numerical models, Balkema, pp. 3-13.

Biarez, J., Hicher, P., 1994. Elementary mechanics of. soil behaviour. Balkema, Rotterdam, The Netherlands, 208 pp.

Bonnard, C., Noverraz, F., Lateltin, O., Raetzo, H., 1995. Large Landslides and Possibilities of Sudden Reactivation. In: Proceedings 44th Geomechanics Colloquy, Salzburg., Austria, pp. 401-407.

Boulahya, F., Dubus, .I.G., Dupros, F., Lombard, P., 2007. Footprint@work, a computing framework for large scale parametric simulations: application to pesticide risk assessment and management, In: Forum EGEE Enabling Grids for E-sciencE, Manchester, UK, pp. 160.

Cressie, N.A.C., 1993. Statistics for Spatial Data, Wiley, New York, 900 pp.

Crosta, G.B., Clague, J.J., 2009. Dating, triggering, modelling, and hazard assessment of large landslides. Geomorphology 103, 1-4.

Dupros, F., Boulahya, F., Vairon, J., Lombard, P., Capit, N., Méhaut, J.F., 2006. IGGI, a computing framework for large scale parametric simulations: application to uncertainty analysis with toughreact. In: Tough symposium 2006, Berkeley, 6 pp.

Evans, S.G., Scarascia Mugnozza, G., Strom, A., Hermanns, R.L., 2002. Landslides from massive rock slope failure. In: Proceedings NATO Advanced Research Workshop on Massive Rock Slope Failure, Celano, Italy, 662 pp. 
van den Ham, G., Rohn, J., Meier, T., Czurda, K., 2009. Finite Element simulation of a slow moving natural slope in the Upper-Austrian Alps using a visco-hypoplastic constitutive model. Geomorphology 103, 136-142.

Hujeux, J.-C., 1985. Une loi de comportement pour le chargement cyclique des sols. In: Davidovici V. (Ed.) Génie Parasismique, Presses ENPC, France, p. 278-302.

Gelman, A., Carlin, J.B., Stern, H.S., Rubin, D.B. 2004. Bayesian Data Analysis, $2^{\text {nd }}$ edn., Chapman and Hall/CRC, London, UK, 698 pp.

Gilks, W., Richardson, S., Spiegelhalter, D. 1996. Markov Chain Monte Carlo in Practice. Chapman and Hall/CRC, London, UK, 512 pp.

Gramacy, R. B., 2007. tgp: An R Package for Bayesian Nonstationary, Semiparametric Nonlinear Regression and Design by Treed Gaussian Process Models. Journal of Statistical Software 19.

Gramacy, R.B., Herbert K.H.L., 2009. Adaptive Design and Analysis of Supercomputer Experiments. Technometrics 51(2), 130-145.

Gramacy, R.B., Taddy, M. 2010. Categorical Inputs, Sensitivity Analysis, Optimization and Importance Tempering with tgp Version 2, an R Package for Treed Gaussian Process Models. Journal of Statistical Software, 33, 1-48.

Gzyl, H., 1995. The Method of Maximum Entropy. In: Bellomo, F., Brezzi, N. (Eds.),Series on Advances in Mathematics for Applied Sciences. World Scientific Publishing Co, p. 29.

Hamm, N.A.S., Hall, J.W., Anderson, M.G., 2006. Variance-based sensitivity analysis of the probability of hydrologically induced slope instability. Computers and Geosciences 32, 803-817.

Hansen, A., 1984. Landslide Hazard Analysis. In: Brunsden, D., Prior, D.B. (Eds.), Slope Instability. Wiley and Sons: New York, pp. 523-602. 
Hastie, T., Tibshirani, R., Friedman, J., 2002. The Elements of Statistical Learning. Springer : Data Mining, Inference, and Prediction, Springer-Verlag, New York, 552pp.

Kennedy, M., O'Hagan, A., 2001. Bayesian calibration of computer models (with discussion). Journal of the Royal Statistical Society 63 (Series B), 425-464.

Koehler, J.R., Owen, A.B., 1996. Computer experiment, In: Ghosh S. and Rao C.R. (Eds.), Handbook of Statistics. Elsevier Science, New York, USA, 13, p. 261-308.

Laloui, L., Tacher, L., Moreni, M., Bonnard, C., 2004. Hydromechanical modeling of crises of large landslides: application to the La Frasse Landslide, In: Proceedings of the $9^{\text {th }}$ International Symposium on Landslides, Rio de Janeiro, Brazil, pp. 1103-1110.

Langewisch, D.R., Apostolakis, G.E., 2010. A comparison of polynomial response surfaces and Gaussian rocesses as metamodels for uncertainty analysis with long-running computer codes. In: Proceedings of the $10^{\text {th }}$ International Probabilistic Safety Assessment \& Management Conference, Seattle, Washington USA, 12 pp.

Le, N.D., Zidek, J.V., 2006. Statistical Analysis of Environmental Space Time Processes. Springer Series in Statistics, New York, 341 pp.

Lopez-Caballero, F., Modaressi Farahmand-Razavi, A., Modaressi, H., 2007. Nonlinear numerical method for earthquake site response analysis —elastoplastic cyclic model and parameter identification strategy. Bulletin of Earthquake Engineering 5(3), 303323.

Lopez-Caballero, F., Modaressi Farahmand-Razavi, A., 2008. Numerical simulation of liquefaction effects on seismic SSI, Soil Dynamics and Earthquake Engineering 28(2) 85-98.

Lophaven, S.N., Nielsen, H.B., Sondergaard J., 2002. DACE—A Matlab kriging toolbox, version 2.0. Technical Report IMM-TR-2002-12, Informatics and Mathematical 
Modelling, Technical University of Denmark, 28 pp. http://www.immm.dtu.dk/_hbn/dace.

750

751

752

753

754

755

756

757

758

759

760

761

762

763

764

765

766

767

768

769

770

771

772

Marrel, A., Iooss, B., Van Dorpe, F., Volkova, E., 2008. An efficient methodology for modeling complex computer codes with Gaussian processes. Computational Statistics and Data Analysis 52, 4731-4744.

Marrel, A., Iooss, B., Laurent B., Roustant O., 2009. Calculations of Sobol indices for the Gaussian process metamodel, Reliability Engineering and System Safety 94, 742-751.

Martin, J.D., Simpson, T.W., 2005. On the use of kriging models to approximate deterministic computer models. Journal of American Institute of Aeronautics and Astronautics 43, 853-863.

McKay, M. D., Beckman R. J. and Conover W. J., 1979. A comparison of three methods for selecting values of input variables in the analysis of output from a computer code. Technometrics 21, 239-245.

Noverraz, F., Bonnard, Ch., 1990. Technical note on the visit of La Frasse Landslide. In: Proceeding $5^{\text {th }}$ International Symposium on Landslides 3, pp. 1549-1554.

Oakley, J.E., O’Hagan, A., 2004. Probabilistic sensitivity analysis of complex models: a Bayesian approach. Journal of Royal Statistical Society 66 (Series B), 751-769.

O’Hagan, A., 2006. Bayesian analysis of computer code outputs: A tutorial. Reliability Engineering and System Safety 91, 1290-1300.

R Development Core Team, 2009. R: A Language and Environment for Statistical Computing. R Foundation for Statistical Computing, Vienna, Austria, 2009. ISBN 3900051-07-0, http://www.R-project.org/

Rasmussen, C. E., 1996. Evaluation of Gaussian Processes and other Methods for Non-linear Regression. PhD thesis, Department of Computer Science, University of Toronto, 132 pp. 
773 Rasmussen, C.E., Williams, C.K.I, 2006. Gaussian processes for machine learning, MIT Press, Cambridge, UK, 266 pp.

Sacks, J., Welch, W.J., Mitchell, T.J., Wynn, H.P., 1989. Design and analysis of computer experiments. Statistical Science 4, 409-435.

Saltelli A., Sobol’ IM., 1995. About the use of rank transformation in sensitivity of model output. Reliability Engineering and System Safety 50, 225-239.

Saltelli A., Chan K., Scott E. M. (Eds.) 2000. Sensitivity Analysis. In: Probability and Statistics Series. Chichester: John Wiley and Sons, 475 p.

Saltelli, A., 2002a. Making best use of model evaluations to compute sensitivity indices. Computer Physics Communications 145, 280-297.

Saltelli, A., 2002b. Sensitivity Analysis for Importance assessment. Risk analysis 22(3), 579590.

Saltelli, A., Ratto, M., Andres, T., Campolongo, F., Cariboni, J., Gatelli, D. Saisana, M., Tarantola, S., 2008. Global sensitivity analysis: The Primer. Wiley, Chichester, UK, $304 \mathrm{pp}$.

Saltelli, A., Annoni, P., Azzini, I., Campolongo, F., Ratto, M., Tarantola, S., 2010. Variance based sensitivity analysis of model output. Design and estimator for the total sensitivity index. Computer Physics Communications 181, 259-270.

Santner, T., Williams, B., Notz, W., 2003. The Design and Analysis of Computer Experiments, Springer Verlag, New York, 308 pp.

Sobol’, I.M., 1993. Sensitivity estimates for non linear mathematical models. Mathematical Modelling and. Computational Experiments 1, 407-414.

Sobol’, I.M. Kucherenko, S.S., 2005. Global sensitivity indices for nonlinear mathematical models, Review of Wilmott Magazine 1, 56-61. 
797 Stein, M.L., 1999. Interpolation of Spatial Data. Springer: Some Theory for Kriging, New $798 \quad$ York, 247 pp.

799 Storlie, C.B., Swiler, L.P., Helton, J.C., Sallaberry, C. J., 2009. Implementation and 800 evaluation of nonparametric regression procedures for sensitivity analysis of 801 computationally demanding models. Reliability Engineering and System Safety 94, $802 \quad 1735-1763$.

803 Von Mises, R., 1964. Mathematical Theory of Probability and Statistics. Mathematical 804 Theory of Probability and Statistics, Academic Press, New York, 694 pp. 


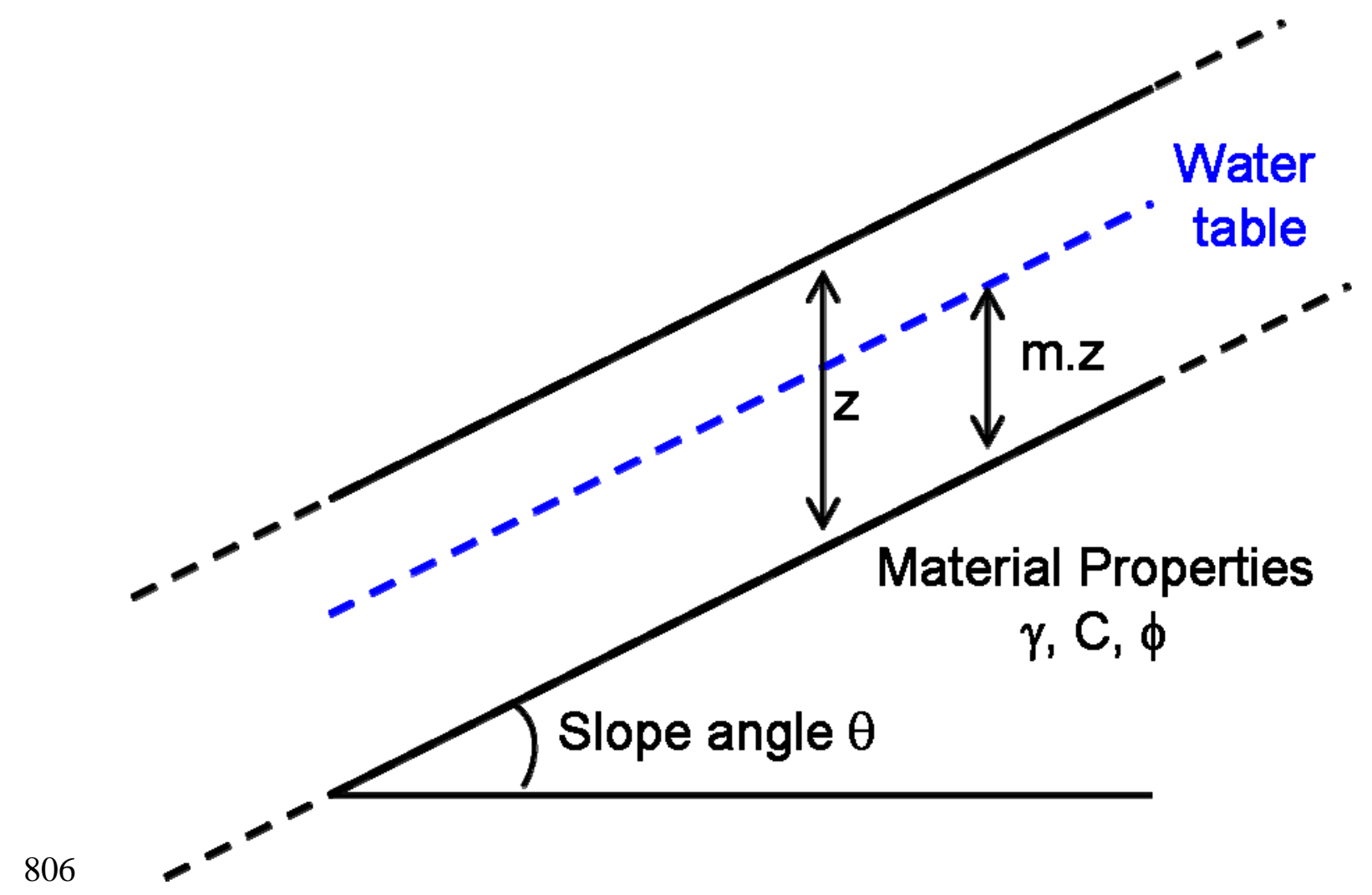

807 Fig. 1: Schematic representation of the infinite slope model (adapted from Hansen, 1984). 
Training sample size of 6
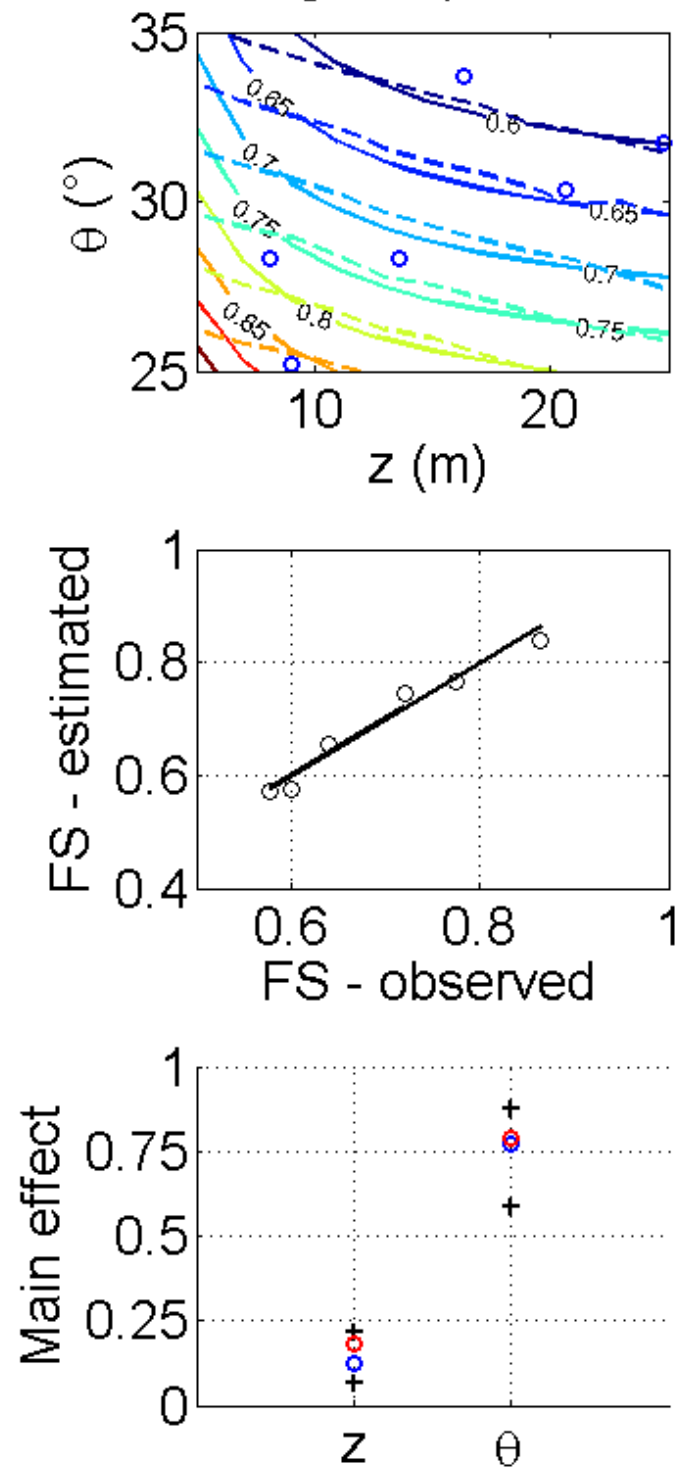

Training sample size of 20
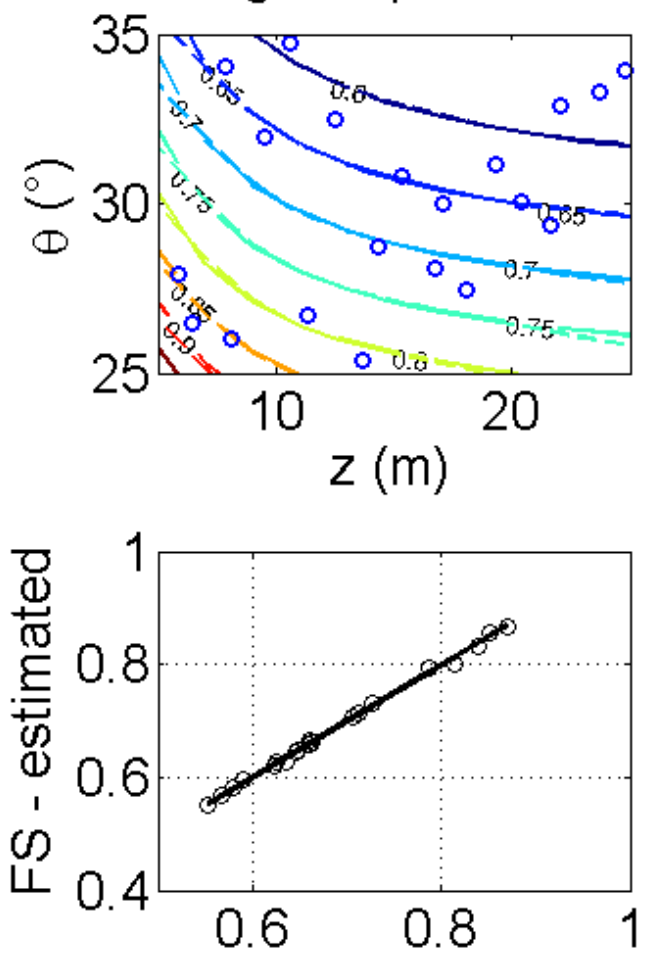

FS - observed

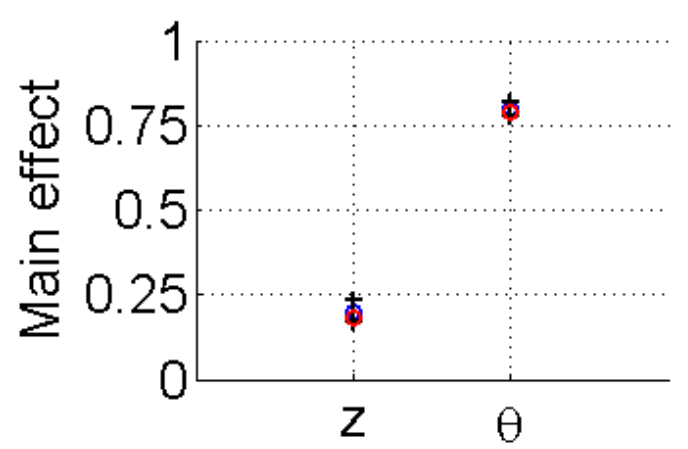

809 Fig. 2: Top: comparison between the true values and the estimates of the factors of safety $F S$ 810 using the GP model constructed with a training sample size of 6 (left) and of 20 (right). The 811 training input configurations are represented by blue dots. Middle: comparison between the 812 observed FS and the estimates within a "leave-one-out” cross validation procedure. Bottom: 813 comparison between the true values (red dots) and the estimates of the main effects for the 814 slope thickness $z$ and of the slope angle $\theta$ (blue dots). The bounds of the confidence intervals 815 associated with both GP models are represented by black cross-type markers. 


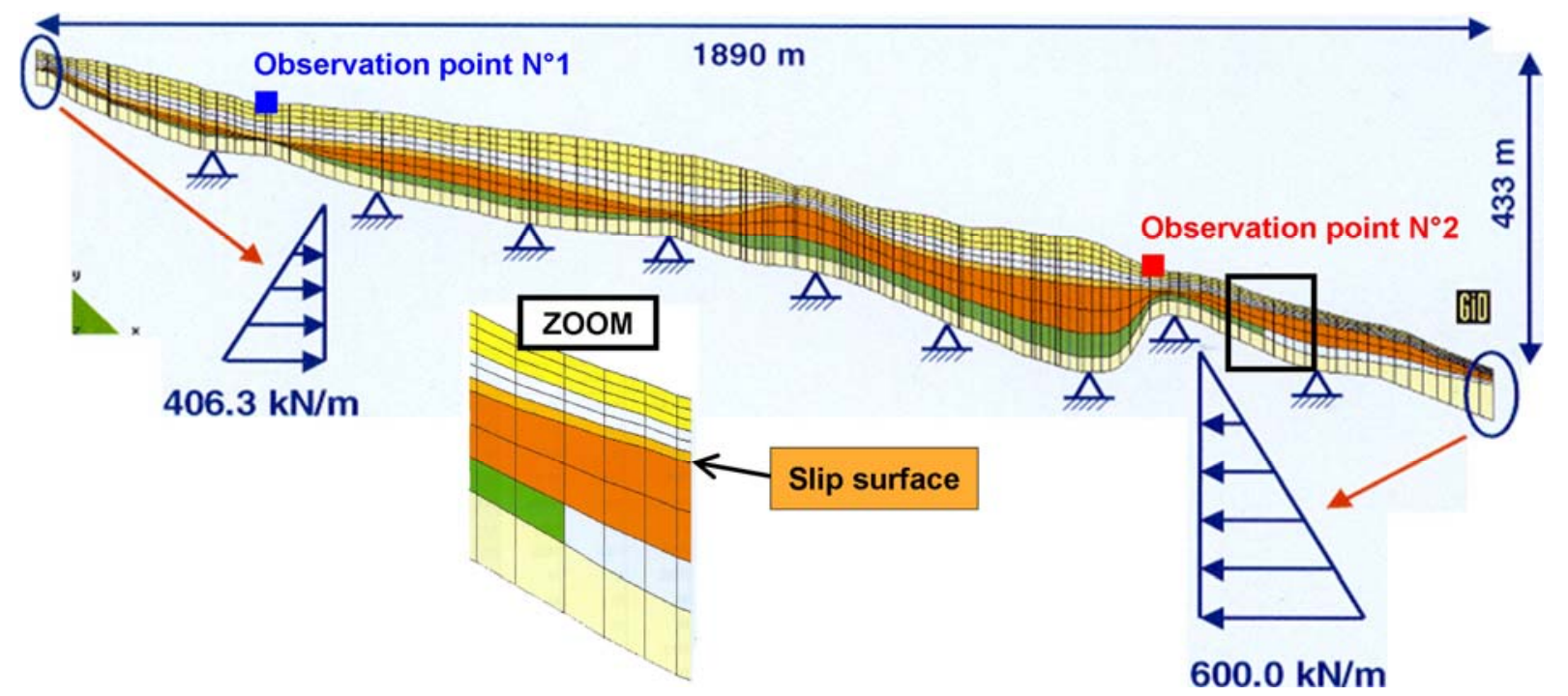

817 Fig. 3: Overview of the landslide numerical model (adapted from Laloui et al., 2004). The

818 slip surface is outlined by the light coloured orange surface. The observation point 1 (in the

819 upper part of the landslide) and observation point 2 (in the lower part of the landslide) used

820 for the sensitivity analysis of the horizontal displacements are respectively outlined by a blue

821 and a red coloured square-type marker.

822
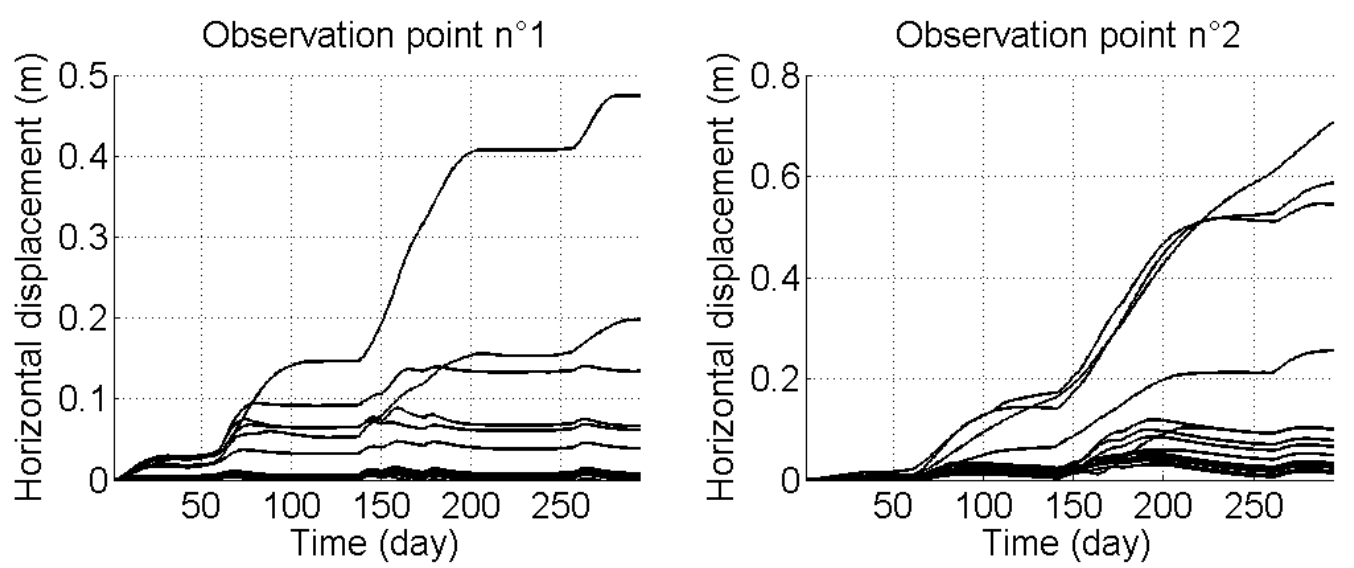

823 Fig. 4: Temporal evolution of the training samples corresponding to the horizontal

824 displacements (m) calculated for 30 different input configurations of the Hujeux law

825 parameters (at the observation point 1 in the upper part of the landslide (left) and at the

826 observation point 2 in the lower part of the landslide (right)). 


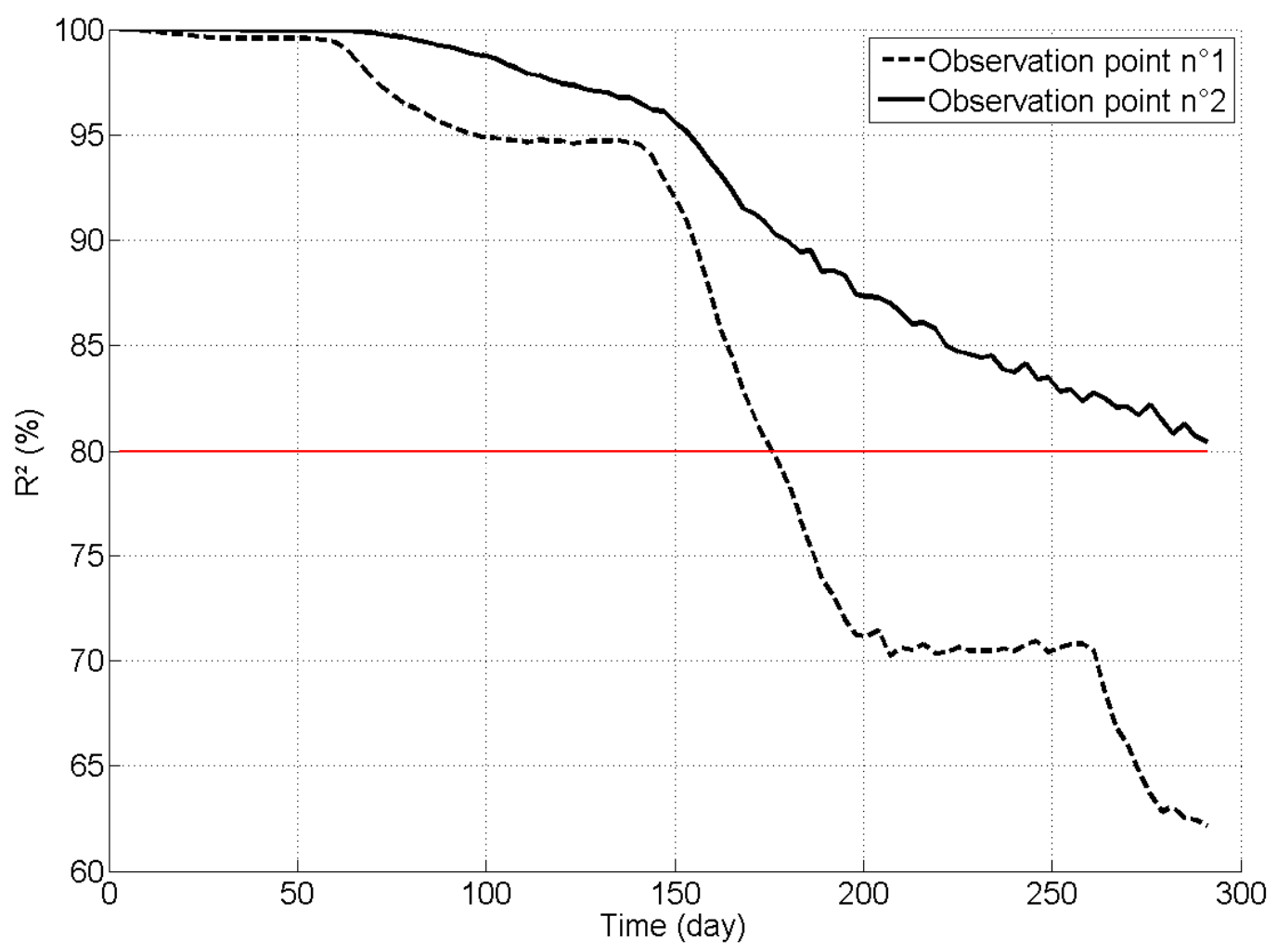

827

828 Fig. 5: Temporal evolution of the coefficient of determination $\mathrm{R}^{2}$ for the "leave-out-out" cross

829 validation procedure of the GP models constructed at each instant of the crisis period at the

830 observation point 1 in the upper part of the landslide (black dashed line) and at the

831 observation point 2 in the lower part of the landslide (black straight line). The threshold of

$83280 \%$ indicating a "satisfactory" predictive quality is outlined by a horizontal red straight line. 

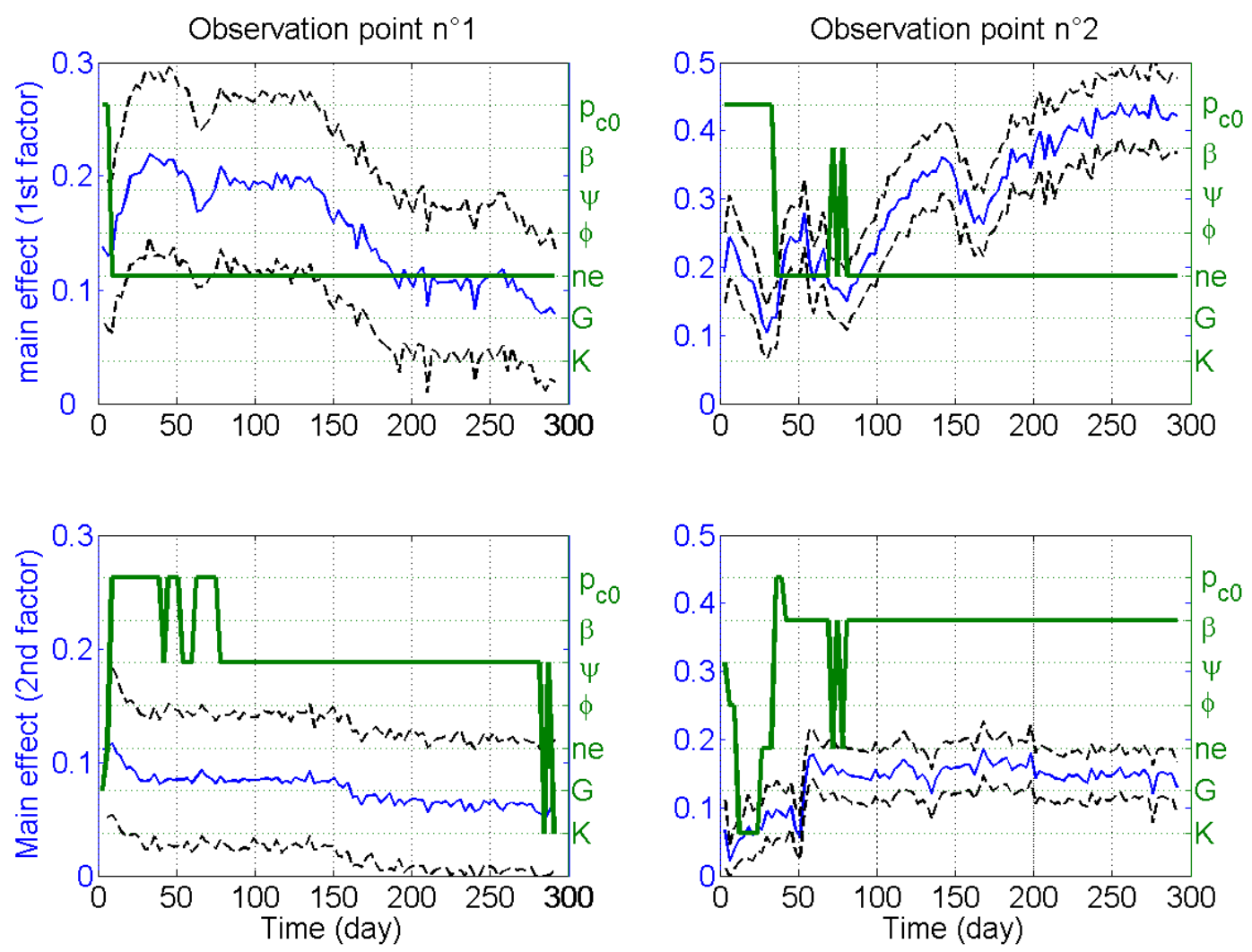

833
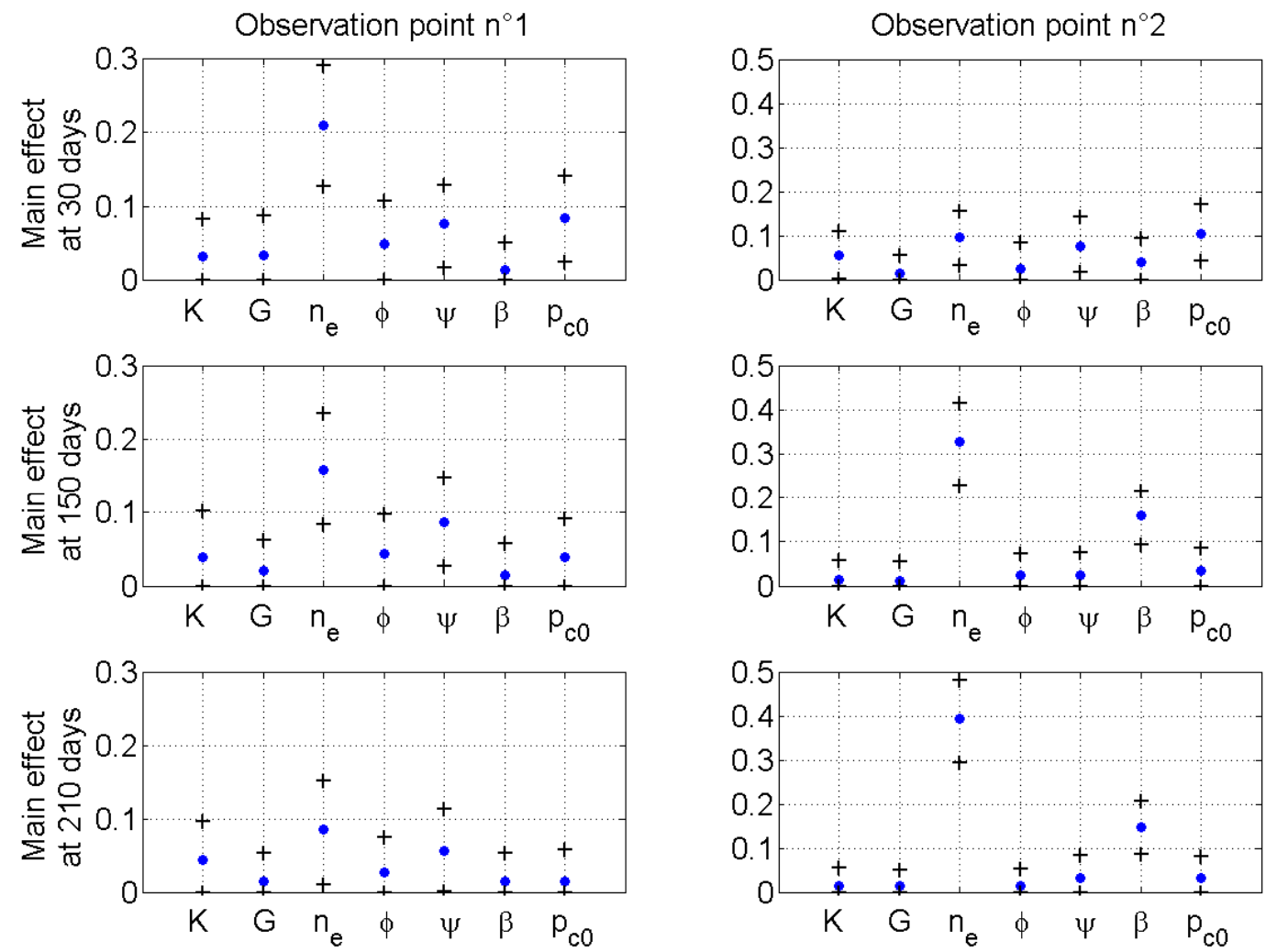

835 Fig. 6: Temporal evolution during the crisis period of the mean of the main effects (blue 836 straight line) at the observation point 1 in the upper part of the landslide (left) and at the 
837 observation point 2 in the lower part of the landslide (right) for the first (green straight line, 838 top) and the second (green straight line, bottom) “most important” input factor. The black 839 dashed lines represent the 5\% and the $95 \%$ quantile.

840

841 Fig.7: Mean of the main effect (blue dots) for each input factor of the slip surface constitutive 842 law at different instants of the crisis period (30 days (top), 150 days (middle) and 210 days 843 (bottom)) at the observation point 1 in the upper part of the landslide (left) and at the 844 observation point 2 in the lower part of the landslide (right). The bounds of the confidence 845 intervals (5\% and $95 \%$ quantile) are outlined by black cross-type markers.

846 
849 Table 1: Comparison between the "true" and the estimates of the main effects for the infinite 850 slope analytical model. $\mu$ corresponds to the mean of the main effect computed with the GP 851 model. $C I$ corresponds to the confidence interval defined by the $5 \%$ and the $95 \%$ quantile 852 computed with the GP model.

\begin{tabular}{|l|l|l|l|}
\hline Input factor & True model & GP model constructed & GP model constructed \\
& & with 6 training samples & with 20 training samples \\
\hline Thickness $z(\mathrm{~m})$ & $18.41 \%$ & $\mu=12.74 \%$ & $\mu=20.02 \%$ \\
& & $C I=[6.48 ; 21.73] \%$ & $C I=[16.90 ; 23.29] \%$ \\
\hline Slope angle $\theta\left(^{\circ}\right)$ & $78.76 \%$ & $\mu=77.01 \%$ & $\mu=79.77 \%$ \\
& & $C I=[58.41 ; 87.43] \%$ & $C I=[77.90 ; 81.68] \%$ \\
\hline
\end{tabular}

853

854 Table 2: Range of values for the slip surface properties of the La Frasse landslide (variation in 855 a range of $25 \%$ around the original values given in Laloui et al., 2004)

\begin{tabular}{|c|c|c|c|c|c|c|c|}
\hline $\begin{array}{l}\text { Input } \\
\text { factor }\end{array}$ & $\begin{array}{l}\text { Vol. } \\
\text { comp. } \\
\text { mod. }\end{array}$ & $\begin{array}{l}\text { Shear } \\
\text { mod. }\end{array}$ & $\begin{array}{l}\text { Non- } \\
\text { linearity } \\
\text { coeff. }\end{array}$ & $\begin{array}{l}\text { Internal } \\
\text { friction } \\
\text { angle }\end{array}$ & $\begin{array}{l}\text { Dilatancy } \\
\text { angle }\end{array}$ & $\begin{array}{l}\text { Plastic } \\
\text { comp. }\end{array}$ & $\begin{array}{l}\text { Initial } \\
\text { critical } \\
\text { pressure }\end{array}$ \\
\hline Symbol & K & G & $\mathrm{n}_{\mathrm{e}}$ & $\phi$ & $\Psi$ & $\beta$ & $\mathrm{p}_{\mathrm{c} 0}$ \\
\hline Unit & $\mathrm{MPa}$ & $\mathrm{MPa}$ & - & $\circ$ & $\circ$ & - & $\mathrm{MPa}$ \\
\hline $\begin{array}{l}\text { Lower } \\
\text { value }\end{array}$ & 180 & 83.25 & 0.225 & 19.125 & 14.25 & 20.625 & 0.375 \\
\hline $\begin{array}{l}\text { Upper } \\
\text { value }\end{array}$ & 300 & 138.75 & 0.375 & 31.875 & 23.75 & 34.375 & 0.625 \\
\hline
\end{tabular}

